\title{
Utilización de un índice clínico-ecográfico para la clasificación de los tumores de ovario
}

\author{
José Saulo Torres R. MD*; Juan Pablo Suso MD**; Eduardo Perea**; Luis Alberto Tafur MD ${ }^{* * *}$; Oswaldo Benítez \\ MD****; Felipe Núñez MD*****
}

\begin{abstract}
RESUMEN:
Diseñar un método que seleccione con cierta confiabilidad entre tumores benignos y malignos, de estos últimos poder seleccionar cuales verdaderamente requieren otros exámenes complementarios o de extensión.
\end{abstract}

PALABRAS CLAVES: Tumores de ovario, Indice ecográfico, Examen físico, Exámenes complementarios.

SUMMARY:

Design a method for a reliable determination of benign and malignant tumors, and be able to decide which malignant tumors actually call for supplementary or additional tests.

KEY WORDS: Ovary tumors, Ultrasound index, Physical Check-up, Supplementary tests.

INTRODUCCION : Considerando que existen criterios clínicos y ecográficos para predecir el comportamiento biológico y la extensión de la enfermedad, se estudió la alternativa de utilizar un puntaje basado en la evaluación clínica y ecográfica de la paciente con diagnóstico presuntivo de tumor de ovario. Se estudiaron 45 pacientes con diagnóstico presuntivo de tumor de ovario entre Enero y Diciembre de 1997

OBJETIVOS : Proponer y evaluar el resultado de la aplicación de un método de predicción mediante el examen físico y la ecografía pélvica, sin estudios de extensión, en pacientes con diagnóstico de tumor de ovario atendidas en el HUV a partir de Enero de 1997.

DISEÑO DEL ESTUDIO: A partir de Enero de 1997 se diseñó un protocolo para ser aplicado en forma prospectiva a todas las pacientes que ingresaban con diagnóstico de tumor de Ovario, el cual incluía entre otros datos: antecedentes personales, síntomas, examen físico e informe ecográfico.

RESULTADOS: Los 45 casos estudiados fueron clasificados histopatológicamente así: benignos 29, de bajo potencial de malignidad 1 malignos 15 . El análisis de los puntajes indica que el índice con valor igual o menor a 7 sin ascitis y movilidad del tumor, permite clasificar los

\footnotetext{
* Jefe sección Ginecología Oncológica H.U.V.

Docente Ad-honorem Universidad del Valle Cali Colombia

** Ginecólogos Oncólogos H.U.V. Docentes Ad-honorem Universidad del Valle

*** Especialista Salud Pública. Profesor Titular Escuela de Salud Pública Universidad del Valle.

Residente de tercer año Universidad Central de Quito. Ecuador.

***** Residente de tercer año Universidad del Valle. Cali-Colombia.
}

tumores benignos con una sensibilidad del 69 (IC :49.0 84.0) y especificidad de 81.3 (IC : 53.7 - 95)

\section{CONCLUSIONES:}

1. Se propone un índice ecográfico con puntaje menor o igual a siete como punto divisorio entre benignidad y malignidad para la clasificación de los tumores ováricos, asociando a este puntaje la ausencia de ascitis y la movilidad del tumor.

2. Las pacientes con un puntaje igual o superior a 8 se proponen solicitar una ecografía abdominal con el fin de evaluar hígado, riñones, sistema pieloureteral y ganglios paraórticos como único examen de extensión.

3. Marcadores Tumorales: Se sugiere tomar muestra para su procesamiento al ingreso y almacenar, cuando la congelación reporte malignidad se envían a proceso. De acuerdo al tipo histologico se seleccionarán el o los marcadores con el fin de disponer de un indicador para el seguimiento posterior.

4. La utilización del Indice clínico-ecográfico permite disminuir los costos del proceso diagnóstico de las pacientes, facilita y agiliza el acceso de las pacientes a la cirugía asegurando su tratamiento.

En dos estudios anteriores, se compararon la biopsia por congelación con la histopatología de los tumores de ovario(1) y se analizó la utilidad de la solicitud de los denominados exámenes de extensión para la predicción del comportamiento biológico y la extensión del tumor $(2,3)$, concluyendo en el primer estudio en la importancia de la biopsia por congelación para la toma de decisiones en el acto quirúrgico y en el segundo la poca 
Cuadro 1

CARACTERISTICAS ECOGRAFICAS. (4)

\begin{tabular}{|l|l|l|l|l|l|}
\hline Puntaje & 0 & \multicolumn{1}{c|}{1} & \multicolumn{1}{c|}{2} & \multicolumn{1}{c|}{3} \\
\hline Volumen & $\leq 10 \mathrm{cc}$ & $\begin{array}{l}10- \\
50 \mathrm{cc}\end{array}$ & $50-200 \mathrm{cc}$ & $200-500 \mathrm{cc}$ & $>500 \mathrm{cc}$ \\
\hline $\begin{array}{l}\text { Grosor de la } \\
\text { pared }\end{array}$ & $\leq 3 \mathrm{~min}$ & $\begin{array}{l}<3 \mathrm{~mm} \text { con } \\
\text { proyecciones } \\
\text { papilares }\end{array}$ & $\begin{array}{l}>3 \mathrm{~mm} \text { con } \\
\text { proyecciones papilares }\end{array}$ & Sólida \\
\hline $\begin{array}{l}\text { Septo } \\
\text { interno }\end{array}$ & No septos & $\begin{array}{l}\text { Delgado } \\
\leq 3 \mathrm{~mm}\end{array}$ & $\begin{array}{l}\text { Grueso 3 - 10 } \\
\mathrm{mm}\end{array}$ & Areas sólidas $>1 \mathrm{~cm}$ & Sólida \\
\hline
\end{tabular}

PUNTAJE TOTAL:

utilidad de los exámenes de extensión y el encarecimiento del proceso diagnóstico.

Considerando que existen criterios a la historia clíni$\mathrm{ca}$, al examen físico y a los hallazgos ecográficos para predecir comportamiento biológico y extensión de la enfermedad, se estudió la alternativa de utilizar un puntaje basado en la evaluación clínica y ecográfica de la paciente con diagnóstico presuntivo de tumor de ovario, cuyos resultados se presentan a continuación.

\section{OBJETIVOS:}

Proponer y evaluar el resultado de la aplicación de un método de predicción mediante la realización del examen físico y la ecografía pélvica, sin mas estudios de extensión, (cistoscopia, rectoscopia, endoscopia vías digestivas altas, urografia, Rx de tórax ) en pacientes con diagnóstico de tumor de ovario atendidas en el HUV a partir de Enero de 1997

\section{MATERIALES Y METODOS:}

Se diseñó un formato (Anexo 1) que incluye datos de la historia clínica, edad, síntomas, algunos datos fundamentales al examen físico y las características ecográficas de la masa en estudio (4).

La ascitis asociada a la masa se consideró como indicio de malignidad.

Tabla 1

\section{DIAGNOSTICO HISTOLOGICO VS PRESUNCION DIAGNOSTICA CUANDO EL INDICE ES $<=7$.}

\begin{tabular}{|l|c|c|c|c|}
\hline $\begin{array}{c}\text { DX DEFINITIVO } \\
\text { PREDICCIÓN? }\end{array}$ & BENIGNO & B.P.M. & MALIGNO & TOTAL \\
\hline BENIGNO & 20 & 1 & 2 & 23 \\
\hline MALIGNO & 3 & 0 & 4 & 7 \\
\hline TOTAL & 23 & 1 & 6 & 30 \\
\hline
\end{tabular}

Tabla 2.

\section{TUMORES DE OVARIO DONDE LA PREDICCION FUE ERRADA CON INDICES $<=7$.}

\begin{tabular}{|c|c|c|c|c|}
\hline No. & Indice & Predicción & Definitivo & Diagnóstico final \\
\hline 126 & 6 & $\mathbf{M}$ & $\mathrm{B}$ & Absceso tuboovarico \\
\hline 145 & 6 & $\mathbf{M}$ & $\mathrm{B}$ & Absceso tuboovarico \\
\hline 147 & 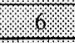 & M & $\mathrm{B}$ & Cistadenoma mucinoso \\
\hline 158 & 16. & B & BPM & $\begin{array}{l}\text { Cistadenoca seroso papilar BPM ovario } \\
\text { izquierdo E IA }\end{array}$ \\
\hline 156 & 1 & B & $M$ & Adenoca mucinoso $\mathrm{G}^{\circ} 1 \mathrm{E} I \mathrm{~A}$ \\
\hline 188 & 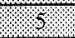 & B & $M$ & Adenoca EndometrioideG ${ }^{\circ} 1 \mathrm{E}$ IA \\
\hline
\end{tabular}

Al evaluar la paciente por primera vez si el Indice ecográfico era igual o menor a 5 , sin ascitis se asumía que el tumor era benigno aunque careciera de movilidad.

Si el índice era mayor a cinco pero menor o igual a 7 , móvil y sin ascitis, se programaba para cirugía asumiéndolo como benigno sin solicitar más exámenes, si carecía de movilidad se predecía maligno ordenando exámenes de extensión.

Si el índice era mayor de siete se asumía como maligno, solicitando exámenes rutinarios de extensión. (6)

Siempre estas decisiones fueron aprobadas en consenso por la junta oncológica.

\section{RESULTADOS:}

Se diligenciaron 45 formularios en pacientes operadas entre enero y diciembre de 1997.

Los informes definitivos de patología de estas pacientes fueron:
Benignos:
29. $(64.4 \%)$
B.P.M:
1. $(2.2 \%)$
Malignos:
15. $(33.3 \%)$
TOTAL
45.

Con índice ecográfico menor o igual a 5 se totalizaron 22 pacientes. Cuando el índice fue de seis se incrementaron a 27 y con índice de siete aumentaron a $30(67 \%)$, discriminados así (ver Tabla 1):

\section{DISCUSION:}

Para predecir el comportamiento biológico de los tumores se utilizaron dos criterios:

1. Una anamnesis adecuada y un examen físico cuidadoso, ya que éstos pueden detectar enfermedad clínica avanzada.

2. La ecografía la que nos proporciona información acerca del tumor respecto a bilateralidad, características internas, superficie capsular, ascitis; adenopatias, medida del endometrio, datos(5) que pueden inducirnos indirectamente hacia un diagnóstico presuntivo de benignidad o malignidad.

El utilizar el índice ecográfico con base en asignar puntajes de acuerdo con el grosor de la pared, septo interno y volumen(3); asociándolo a ascitis, y movilidad, se propone una clasificación prequirúrgica presuntiva del comportamiento biológico del tumor

El análisis de los puntajes indica que el índice con valor igual o menor de siete permite clasificar los tumo-

Tabla 3

TUMORES DE OVARIO BENIGNOS CON PUNTAJE ECOGRAFICO SUPERIOR A 7

\begin{tabular}{|c|c|c|c|c|}
\hline $\mathrm{N}^{\circ}$ & Indice & 41001006 & Definitivo & DIAGNOSTICO \\
\hline 124 & 10 & 8 & $\mathrm{~B}$ & Teratoma Quístico Maduro. \\
\hline 129 & 8 & \% & $\mathrm{B}$ & Cistadenoma mucinoso \\
\hline 137 & 8 & X & $\mathrm{B}$ & Endometrioma abscedado \\
\hline 140 & 12 & M & $\mathrm{B}$ & Fibroma Ovárico \\
\hline 142 & 10 & 6 & $\mathrm{~B}$ & Tecoma \\
\hline 197 & 11 & W & $\mathrm{B}$ & Tecoma \\
\hline
\end{tabular}


res benignos con una sensibilidad de 69.0(IC: 49.0 - 84.0) y una especificidad de 81.3(IC: 53.7 - 95) comparando con el diagnóstico histopatológico definitivo. De esta manera quedaron incluidos 30/45 tumores y combinándolo con las variables movilidad y ascitis se acertó adecuadamente en 20/23 (87\%) de los benignos, 4/7 $(57 \%)$ de los malignos y en 6 no se acertó (Ver Tabla 2.).

De los catalogados benignos 3 fueron malignos pero Estadios IA, lo que significó ganancia al no haberles solicitado exámenes de extensión. Tres fueron catalogados como malignos, dos se programaron como urgencias con diagnóstico clínico de Tumores Ovaricos abscedados y el tercero se estudió solicitándole todos los exámenes de extensión.
Tabla 4

\section{TUMORES DE OVARIO HISTOLOGICAMENTE MALIGNOS CON PUNTAJE ECOGRAFICO SUPERIOR A 7}

\begin{tabular}{|c|c|c|c|c|}
\hline Cod & Indice & ledicerin & Definitivo & DIAGNOSTICO \\
\hline 116 & 8 & 13 & $\mathrm{M}$ & $\begin{array}{l}\text { Cistadenoca metastásico seroso papilar-pelvis } \\
\text { congelada E IV }\end{array}$ \\
\hline 117 & 10 & M & M & Tumor Células de la granulosa E IA \\
\hline 120 & 8 & 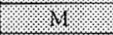 & M & Carcinoma $\mathrm{G}^{\circ} 3$ Bilateral Ovario IIIB \\
\hline 121 & 9 & 唯 & $\mathrm{M}$ & Cistadenoca mucinoso $\mathrm{G}^{\circ} 2$. E IIIB \\
\hline 131 & 8 & 稵 & $M$ & Tumor de células de la granulosa bilateral E IV \\
\hline 134 & 10 & M & $M$ & Carcinosarcoma E IIIC \\
\hline 189 & 9 & 器 & $\mathrm{M}$ & Neurofibrosarcoma metastásico EIUB \\
\hline 192 & 8 & \% & $M$ & Adenoca papilar metastásico E IVB \\
\hline 201 & 8 & X & M & Adenoca papilar metastásico E IVB \\
\hline
\end{tabular}

\section{CUESTIONARIO DE INVESTIGACION (ANEXO 1.)}

Nombre: Historia Clínica: $\mathrm{N}^{\circ}:$

\begin{tabular}{|c|c|}
\hline A: & $\begin{array}{l}15-20() \\
21-29()\end{array}$ \\
\hline
\end{tabular}

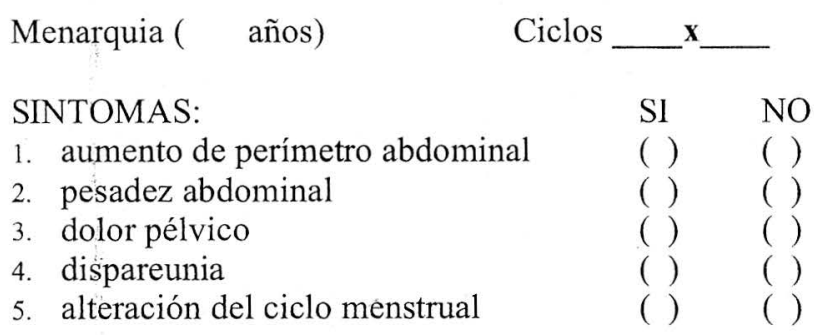

\section{EXAMEN FÍSICO}

1. localización masa

2. movilidad

3. consistencia quistica

4. tamaño

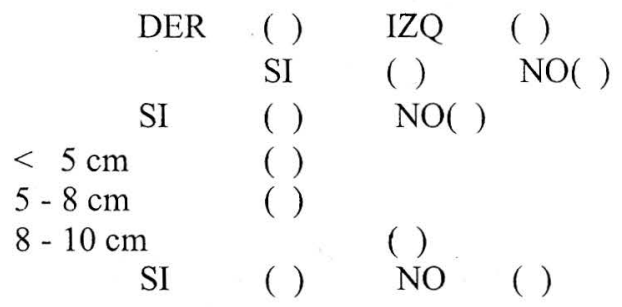

5.ascitis SI

CARACTERÍSTICAS ECOGRAFICAS.

Cuadro 1. Puntaje para las características ecograficas de masa ovárica.

\begin{tabular}{|l|l|l|l|l|l|}
\hline & 0 & \multicolumn{1}{c|}{1} & \multicolumn{1}{c|}{2} & \multicolumn{1}{c|}{3} & \multicolumn{1}{c|}{4} \\
\hline Volumen & $\leq 10 \mathrm{cc}$ & $10-50 \mathrm{cc}$ & $50-200 \mathrm{cc}$ & $200-500 \mathrm{cc}$ & $>500 \mathrm{cc}$ \\
\hline $\begin{array}{l}\text { Grosor de la } \\
\text { pared }\end{array}$ & $\leq 3 \mathrm{~mm}$ & $\begin{array}{l}<3 \mathrm{~mm} \text { con } \\
\text { proyección } \\
\text { pailares }\end{array}$ & $\begin{array}{l}>3 \mathrm{~mm} \text { con } \\
\text { proyección papilares }\end{array}$ & Sólida \\
\hline $\begin{array}{l}\text { Septo } \\
\text { interno }\end{array}$ & No septos & $\begin{array}{l}\text { Delgado } \\
\leq 3 \mathrm{~mm}\end{array}$ & $\begin{array}{l}\text { Grueso } \\
3-10 \mathrm{~mm}\end{array}$ & Areas sólidás $>1 \mathrm{~cm}$ & Sólida \\
\hline
\end{tabular}

PUNTAJE TOTAL:

HISTOPATOLOGICO: 
En resumen se dejaron de solicitar 23/30 exámenes de extensión por catalogarse benignos, los restantes 2 ingresaron por urgencias siendo operados como tales y solo a 5 catalogados malignos se les solicito exámenes de extensión.

Aquellos con índice mayor de 7 todos fueron catalogados como malignos a excepción de uno que por las calcificaciones reportadas a la ecografia se catalogo como teratoma, ya que estos en el $99 \%$ de los casos son benignos (7). ( Ver Tabla No3.)

De los 29 clasificados histológicamente como benignos, 6 no se incluyeron, ya que presentaron un índice mayor a 7. Corresponden 3 al grupo fibro-tecoma que ecográficamente son sólidos, razón por la cual se les asigna un puntaje mayor, los otros dos correspondían a un endometrioma abscedado y a un cistadenoma mucinoso de gran tamaño, y el último fue acertadamente catalogado como benigno, ya que presentaba calcificaciones en su interior interpretándose como teratoma.

De los 15 casos de tumores malignos, ya analizamos 6 que fueron mal catalogados benignos, los 9 restantes fueron acertadamente señalados como malignos, a todos se les solicitaron exámenes de extensión, ninguno de los cuales aportó información adicional. ( Ver Tabla 4.)

Teniendo en cuenta que el puntaje igual o superior a 8 nos orienta hacia el diagnóstico de malignidad, consideramos importante evaluar todo el abdomen solicitando una ecografía abdominal para evaluar hígado, riñones, sistema pieloureteral y ganglios paraórticos.

El marcador tumoral en nuestra institución de rutina se toma en la sala de hospitalización previa a la cirugia, la muestra se almacena refrigerada y en caso que la biopsia por congelación sea positiva se envía a proceso con el fin de disponer de un indicador para seguimiento posterior de la enfermedad. Esta medida evita incurrir en costos innecesarios.

Con base en nuestros resultados proponemos el examen clínico y el índice ecográfico como únicas evaluaciones prequirurgicas para predecir el comportamiento biologico del tumor tomando como puntaje divisorio entre benigno y maligno el valor igual o menor de 7 asociando ascitis y movilidad.

\section{CONCLUSIONES}

1. Se propone utilizar un índice clínico-ecográfico para la clasificación de los tumores ováricos utilizando como punto divisorio entre benignidad y malignidad el puntaje ecografico hasta 7 , asociado a la variable ascitis y movilidad.

2. A las pacientes con un puntaje igual o superior a 8 se propone solicitar una ecografía abdominal con el fin de evaluar hígado, riñones, sistema pieloureteral y ganglios paraórticos.

3. Se sugiere tomar muestra para marcador tumoral antes de la cirugía y en caso de que la congelación sea positiva enviarlo a procesar con el fin de disponer de un indicador para el seguimiento posterior de la enfermedad en la paciente.

4. La utilidad de estas recomendaciones se relaciona con disminución de costos, acceder rápidamente al tratamiento quirúrgico y descongestión de otros servicios al no remitirse para exámenes seguramente innecesarios.

\section{BIBLIOGRAFIA}

1. Torres J S, Suso JP, Perea E, Tafur LA, Agudelo, M. Tumores ováricos: Correlaciòn entre los informes de estudios solicitados por congelación y la histopatología definitiva. HUV. 1994 -1997. Rev Col Obst. Ginec 1998.

2. Peter G Rose, MD, Ronald B. Rubin, MD, Beth E. Nelson, MD, Richard E. Hunter,MD and Frank R. Reale, MD. Accuracy of frozensection (intraoperative consultation) diagnosis of ovarian tumors. Worcester, Massachutts.September. Am J Obstet Gynecol 1994;171:823-6.

3. Torres J/S, Suso JP, Perea E, Tafur LA, Fernández R. Tumores de ovario. Exámenes de extensión. ¿ una medida exagerada ? Experiencia en el HUV. . Rev Col Obst. Ginec 1998.
4. DePriest PD, Shenson-D,Fried-A; Hunter-JE; Andrews-SJ; Gallion HH; Pavlik-EJ;Kryscio-RJ; Van Nagell-JR Jr.Department of Obstetrics and Gynecology, University of Kentucky Medical Center,Lexington. A morfology index based on sonographyc findings in ovarian cancer. Gyncol oncology 1993 Oct; 51:7-11.

5. Protocolos de manejo en patología gineco-oncologica. H.U.V 1995.

6. Callen. Ecografia en Obstetricia y Ginecología. Evaluación ecografica del ovario. Pag.436. Editorial Panamericana, Buenos Aires Argentina.1993.

7. Piver Steven M. Handbook of Gynecologic Oncology. $2^{\text {nd }}$ ed. 1996. Ovarian germ cell tumors. pag 41 Little, Brown and Company, New York. 